\title{
Development of a space spectropolarimeter for full stokes parameters retrieval
}

Vasilescu, Bogdan, Nazé, Yaël, Rauw, Grégor, Kintziger, Christian, Loicq, Jérôme

Bogdan Vasilescu, Yaël Nazé, Grégor Rauw, Christian Kintziger, Jérôme Loicq, "Development of a space spectropolarimeter for full stokes parameters retrieval," Proc. SPIE 11852, International Conference on Space Optics ICSO 2020, 118522J (11 June 2021); doi: 10.1117/12.2599427

SPIE Event: International Conference on Space Optics - ICSO 2021, 2021, Online Only 


\section{International Conference on Space Optics-ICSO 2020}

Virtual Conference

30 March-2 April 2021

Edited by Bruno Cugny, Zoran Sodnik, and Nikos Karafolas
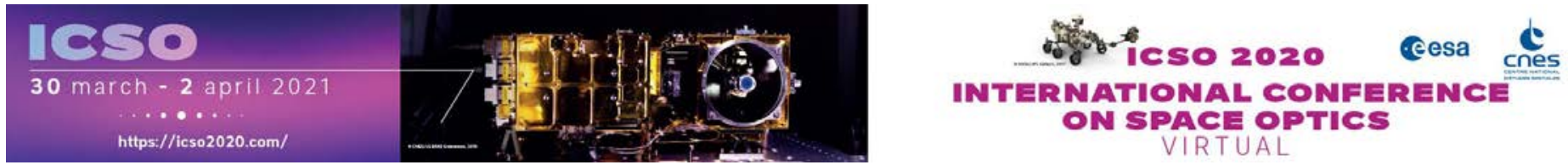

\section{Development of a space spectropolarimeter for full stokes parameters retrieval}

\section{Cesa iso poresedings ecnes}




\title{
Development of a space spectropolarimeter for full Stokes parameters retrieval
}

\author{
Bogdan Vasilescu ${ }^{\mathrm{a}}$, Yaël Nazéb ${ }^{\mathrm{b}}$, Grégor Rauw ${ }^{\mathrm{c}}$, Christian Kintziger $^{\mathrm{d}}$, and Jérôme Loicq ${ }^{\mathrm{a}}$ \\ aUniversité de Liège, Centre Spatial de Liège, Avenue du Pré Aily, Liège, Belgiume, 4031 \\ ${ }^{\text {b} F N R S ~ S e n i o r ~ R e s e a r c h e r, ~ U n i v e r s i t e ́ ~ d e ~ L i e ̀ g e, ~ A l l e ́ e ~ d u ~ S i x ~ A o u ̂ t, ~ 19 C, ~ L i e ̀ g e, ~ B e l g i u m, ~} 4000$ \\ ${ }^{\mathrm{c}}$ Université de Liège, Allée du Six Août, 19C, Liège, Belgium, 4000 \\ ${ }^{\mathrm{d} C e n t r e ~ S p a t i a l ~ d e ~ L i e ̀ g e, ~ A v e n u e ~ d u ~ P r e ́ ~ A i l y, ~ L i e ̀ g e, ~ B e l g i u m e, ~} 4031$
}

\begin{abstract}
This paper presents the firsts steps of the development of a new spectropolarimeter with a high potential for space applications. The instrument, designed on birefringent elements, avoids the need for rotating elements and may cover the entire Stokes vector on a large wavelength band. In addition, the architecture of the modulator enables a very high polarimetric efficiency, placing this concept above classical spectropolarimeters. A new proof for the solution uniqueness is presented, and base on that, a thorough analysis of the polarimetric efficiency.
\end{abstract}

Keywords: polarimetry, spectropolarimetry, birefringence, space optics, polarimetric modulation

\section{INTRODUCTION}

The polarization is a valuable scientific mean for the investigation of the medium encountered by light on its path. The applications are almost countless today, ranging from biology and medicine and to astronomy or remote observation of the Earth. Therefore, in astronomy, the polarization can be used to evaluate the magnetic field of stars, the distribution of the interstellar medium, or to model the structure of the upper layer of remote objects, like the asteroids, only to cite a few of its applications. ${ }^{1-3}$ Because most of the optical detectors are insensitive to the orientation of the electric field of light, the measurement of the polarization remains a challenging task. In principle, in order to access this property, the different states of polarization must be converted into corresponding different intensities. To do so, most of the polarimeters are using a modulator, that converts a precise type of incoming polarization into defined series of other types of polarization. With the help of an analyzer then, an intensity depending on these types of polarization can be recorded.

In practice, two significant families of measurement techniques have been developed to determine the polarization of light: one using rotating components and another using amplitude division. Both procedures occupy a relatively large volume and make use of complex mechanisms. The considerable dimensions and the need for rotating parts are the most significant drawbacks for space usage as they directly impact the cost, the design, and the reliability of space missions.

A new method for single-shot full Stokes polarimetry is proposed by Vasilescu et al. ${ }^{4}$ With an extremely compact and robust design, the new method avoids the use of movable components or the classical division of amplitude. The modulator of this new type of instrument is able to introduce a continuous modulation of the received signal in the vertical direction. Given the dependency on the wavelength of the birefringence of the modulator, the induced modulation is also wavelength-dependent. Therefore, using an appropriate material for the modulator, a large wavelength range can be covered. For instance, the use of magnesium fluoride $\left(\mathrm{MgF}_{2}\right)$ gives access to the entire spectrum between 0.12 and $7 \mu \mathrm{m}$, whereas with calcium fluoride $\left(\mathrm{CaF}_{2}\right)$ the covered window is even larger, from 0.13 to $9.7 \mu \mathrm{m}$. The only limitation is coming then from the working range of the detector. To be complete, a spectrometer needs to be added after the modulator. The general layout is presented in Fig. 1.

Further author information: (Send correspondence to Bogdan Vasilescu)

Bogdan Vasilescu: E-mail: bvasilescu@uliege.be 


\section{SPECTROPOLARIMETER CONCEPT}

The key part of the studied concept of the instrument is the modulator. It is made of three birefringent uniaxial elements of the same material, such as $\mathrm{MgF}_{2}$ or $\mathrm{CaF}_{2}$. Because $\mathrm{MgF}_{2}$ is used on a broader scale in the manufacturing of optical components, the corresponding birefringence data were used during our simulations. A schematic representation of the modulator can be seen in Fig. 1. This part is composed of three prisms assembled together in a compact structure. The first wedge has a very small apex angle $\left(\xi<3^{\circ}\right)$, and an optical fast axis oriented along the $x$-axis. Given the continuous variation of the optical path distance with the vertical position of the incoming rays, all the rays will acquire a continuous phase variation in the vertical direction by passing through this prism. The second element has the fast optical axis oriented along the $z$-axis (following the direction of propagation of light), and therefore has no impact upon the phase. In the third element, having an apex angle $\psi$, the fast axis is oriented at $45^{\circ}$, with respect to the $x$-axis, in the $x y$ plane. Therefore, the third element will introduce a supplementary phase difference between the components of light, depending also on the position on the vertical. A supplementary modulation of the incoming polarization is then produced.

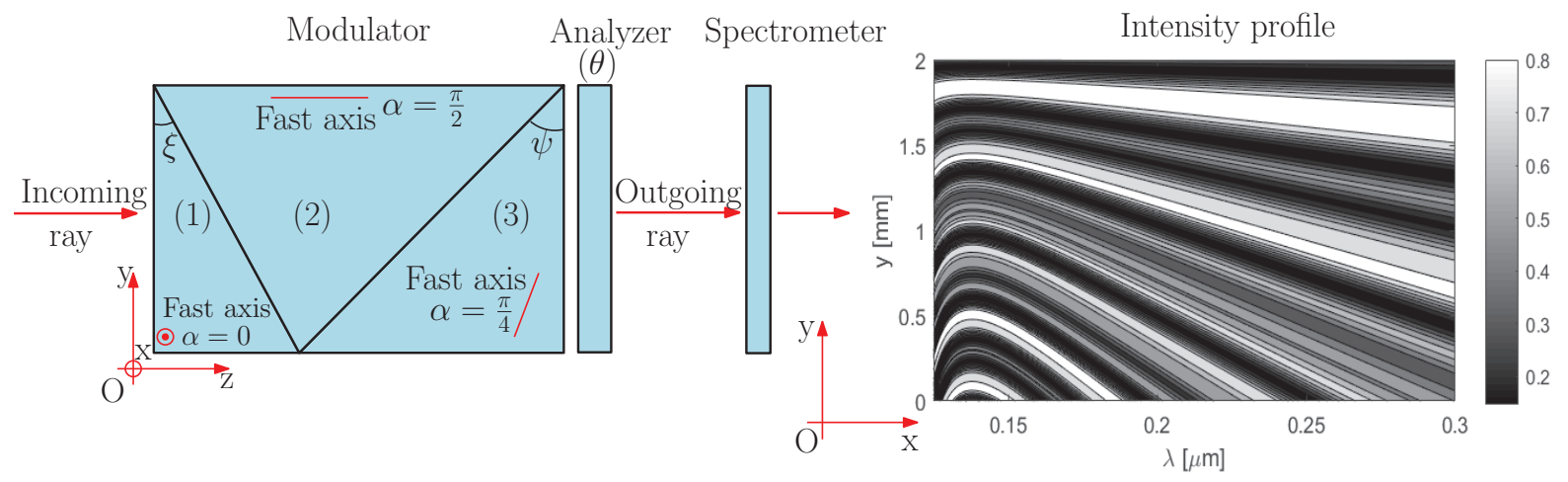

Figure 1. The collimated incoming light arriving from the left-hand side is perpendicular to the surface of the modulator, the ( $x y$ plane). After passing through the polarimeter, the emerging light is modulated along the vertical direction (along the $y$-axis). The spectrometer then leads to a wavelength dispersion along the horizontal direction ( $x$-axis). The observed intensity profile represented here corresponds to an arbitrary state of polarization $\mathbf{S}=[1,0.4,0.3,0.5]^{T}$ and to a modulator build in $\mathrm{MgF}_{2}$, with the apex angles $\xi=1.5^{\circ}$ and $\psi=3^{\circ}$. The orientation of the analyzer was $\theta=90^{\circ}$. Additionally, it was considered that the entire incoming beam is identically polarized, being characterized by a single polarization state, i.e. the vector $\mathbf{S}$.

The reasons for this complex structure can be easily understood with the help of the Poincaré spheres. These allow the representation of the Stokes vectors of polarized light, $S=[I, Q, U, V]^{T}$, on a sphere, in the space $(Q, U, V)$. The radius of this sphere is given by the degree of polarization, $\left.p=\sqrt{(} Q^{2}+U^{2}+V^{2}\right) / I$. The normalized Stokes parameters, $Q / I, U / I, V / I$, are associated then to o single point on the surface of the sphere.

Therefore, given the orientation of the fast axis inside the first wedge, any incoming state of polarization will be converted by this first element into polarization states in which the $Q$ term of the Stokes vector is not modulated. The states of polarization emerging from the first wedge at different locations along the vertical direction are situated on the Poincaré sphere in a plane parallel to the $(U V)$ plane (Fig. 2-left). Therefore, the first element of the modulator is not enough to retrieve the $Q$ parameter. Adding a second wedge (element (3) in Fig. 1), with the fast axis oriented at $45^{\circ}$ with respect to the $x$-axis, in $x y$-plane, ensures a complete modulation of any incoming state of polarization (Fig. 2-right). At any point in the vertical direction on the exit plane of the modulator different states of polarization are registered, as can be observed in Fig. 2-right. This pattern of the outgoing polarization, implemented on the Poincaré sphere, ensures a complete determination of the observed polarization as the projections along the $Q, U$ and $V$ axis are continuously varying with the vertical position. 

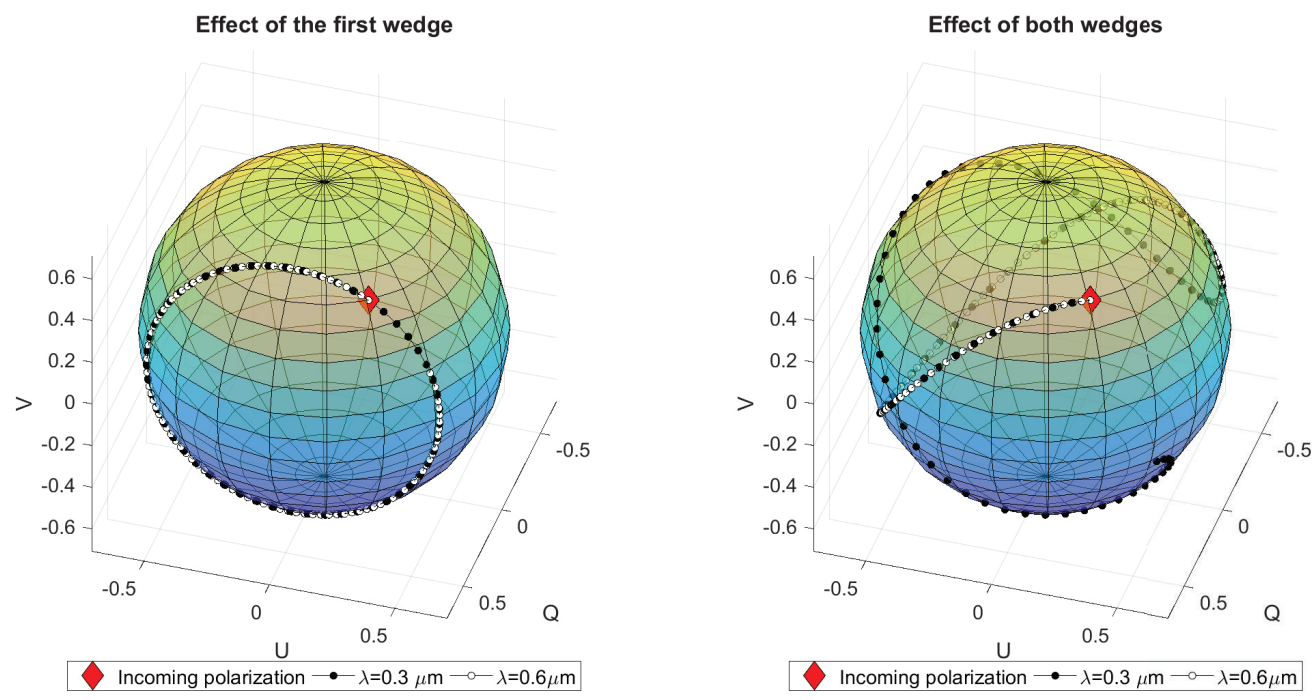

Figure 2. Poincaré representation of the modulation produced by the first wedge alone (left-side), and by the entire modulator (at right). The red square represents an arbitrary incoming state of polarization $\left(\mathbf{S}=[1,0.4,0.3,0.5]^{T}\right)$, while the black and white dots correspond to the outgoing states of polarization at different positions in the vertical direction, for two values of the wavelength. It can be noticed that the first wedge lets the $Q$ parameter undetermined. The outgoing polarization stays in a plane parallel to the $(U V)$ plane and the $Q$ projection is constant. Nevertheless, adding the element (3) (see Fig. 1) helps to achieve a complete modulation for $Q, U$, and $V$.

Apart from the modulator, the polarimeter uses an analyzer oriented at an angle $\theta$ about the $x$ axis, in the plane $(x y)$. After the passage through the polarimeter, the light is spectrally dispersed by the spectrometer over the $x$-axis.

Using the Stokes formalism, which describes the polarization with the help of a vector with four parameters, $\left(\mathbf{S}=[I, Q, U, V]^{T}\right)$, and the Mueller calculus, the light passing through this modulator receives a modulation expressed as:

$$
I_{\text {out }}(\theta, y, \lambda)=\frac{1}{2}\left(I_{\text {in }}+Q_{\text {in }} \cdot m(\theta, y, \lambda)+U_{\text {in }} \cdot n(\theta, y, \lambda)+V_{\text {in }} \cdot p(\theta, y, \lambda)\right)
$$

where

$$
\left\{\begin{array}{l}
m(\theta, y, \lambda)=\cos (2 \theta) \cos \Delta \phi_{3} \\
n(\theta, y, \lambda)=\sin (2 \theta) \cos \Delta \phi_{1}+\cos (2 \theta) \sin \Delta \phi_{1} \sin \Delta \phi_{3} \\
p(\theta, y, \lambda)=\cos (2 \theta) \cos \Delta \phi_{1} \sin \Delta \phi_{3}-\sin (2 \theta) \sin \Delta \phi_{1}
\end{array}\right.
$$

and

$$
\left\{\begin{array}{l}
\Delta \phi_{1}=\frac{2 \pi}{\lambda} \Delta n(\lambda)(h-y) \tan \xi \\
\Delta \phi_{3}=\frac{2 \pi}{\lambda} \Delta n(\lambda)(h-y) \tan \psi,
\end{array}\right.
$$

where $\Delta n(\lambda)=\left|n_{o}(\lambda)-n_{e}(\lambda)\right|$ is the birefringence of the material, $h$ is the height of the modulator, and $\mathbf{S}_{\text {in }}=\left[I_{i n}, Q_{i n}, U_{i n}, V_{i n}\right]^{T}$ is the incoming Stokes vector.

Fitting the function (1) with the data from the detector plane, the Stokes parameters can be inferred. 


\section{UNIQUENESS OF THE SOLUTION AND HIGH EFFICIENCY}

\subsection{Uniqueness of the solution}

In order to ensure the measurement of polarization, the spectropolarimeter must provide a unique Stokes vector $\mathbf{S}_{\text {in }}=\left[I_{i n}, Q_{i n}, U_{i n}, V_{i n}\right]^{T}$ for each pattern of intensity from the detector plane. Using the theorem of Wronskian it has been proven previously that this solution is unique. ${ }^{4}$ The same proof can be obtain in a faster way by testing the orthogonality of the modulation functions $1, m(y), n(y)$ and $p(y)$, corresponding to the Stokes parameters $I_{i n}, Q_{i n}, U_{i n}$ and $V_{i n}$ (Eq.1). Theoretically, two functions, $f(x), g(x)$ are orthogonal on the interval $[a, b]$ if

$$
<f, g>=\int_{a}^{b} \overline{f(x)} g(x) d x=0
$$

If $(1, m(y), n(y), p(y))$ are orthogonal, then they form a base over the considered interval and, consequently, the decomposition of $S_{i n}$ on this base is unique. To a given incoming Stokes vector will correspond a unique pattern of the intensity on the detector plane. Fig. 3 shows that, given the periodic character of the modulation functions, the scalar product has also a periodic behavior. For the case of $\lambda=0.3 \mu \mathrm{m}$, presented in the Fig. 3, the period is $[0,6.9] \mathrm{mm}$. Therefore, the modulation functions $(1, m(y), n(y), p(y))$ are orthogonal over the intervals $[0, n \cdot 6.9] \mathrm{mm}, n \geq 1$, thus over the height of the modulator also, of approximately $1 \mathrm{~cm}$. Consequently, the decomposition of the incoming Stokes vector is unique over this distance. Changing the wavelength affects only the period of the scalar product, but not the periodic character. Thus, the orthogonality is true for any wavelength in the considered window of the modulator.

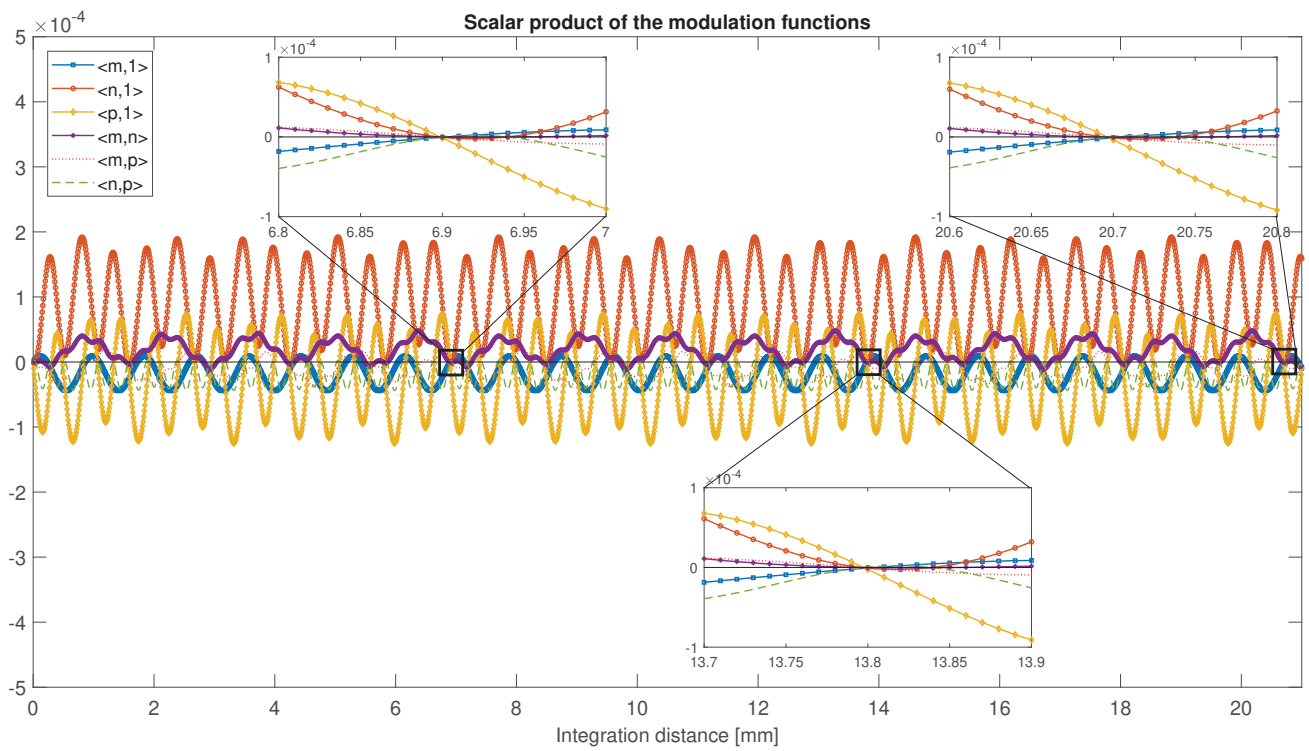

Figure 3. Variation of the scalar product of the modulation functions $(1, m(y), n(y), p(y))$, with the integration distance. It can be noticed that for an integration over $[0,6.9] \mathrm{mm},[0,13.8] \mathrm{mm},[0,20.7] \mathrm{mm}$, for arbitrary angles $\theta=50.6^{\circ}, \xi=2.6^{\circ}$, $\psi=1.6^{\circ}$, and $\lambda=0.3 \mu \mathrm{m}$, the scalar product is zero (zoomed regions aside the main graphic).

\subsection{Efficiency of the modulation scheme}

One of the easiest ways to assess the performance of a polarimeter is through the mean of the efficiency of the modulation scheme (i.e. Eq. (1) written for a column of $N$ pixels), which is inversely proportional to the uncertainty affecting the retrieval of the Stokes parameters. ${ }^{5}$ Writing the equation (1) for a column of $N$ pixels, we obtain the system: 


$$
\underbrace{\left(\begin{array}{c}
I_{\text {out }}\left(y_{1}\right) \\
I_{\text {out }}\left(y_{2}\right) \\
\ldots \ldots \\
I_{\text {out }}\left(y_{N}\right)
\end{array}\right)}_{\mathbf{I}_{\text {out }}}=\frac{1}{2} \underbrace{\left(\begin{array}{cccc}
1 & m\left(y_{1}\right) & n\left(y_{1}\right) & p\left(y_{1}\right) \\
1 & m\left(y_{2}\right) & n\left(y_{2}\right) & p\left(y_{2}\right) \\
\ldots & \ldots & \ldots & \ldots \\
1 & m\left(y_{N}\right) & n\left(y_{N}\right) & p\left(y_{N}\right)
\end{array}\right)}_{\mathbf{O}} \cdot \underbrace{\left(\begin{array}{c}
I_{\text {in }} \\
Q_{\text {in }} \\
U_{\text {in }} \\
V_{\text {in }}
\end{array}\right)}_{\mathbf{S}_{\text {in }}},
$$

or

$$
\mathbf{I}_{\mathrm{out}}=\mathbf{O} \cdot \mathbf{S}_{\mathrm{in}},
$$

where $\mathbf{O}$ is the modulation matrix. Therefore

$$
\mathbf{S}_{\text {in }}=\mathbf{O}^{-1} \cdot \mathbf{I}_{\text {out }}
$$

where $\mathbf{O}^{-1}$ is the demodulation matrix (also written as $\mathbf{D}$ ).

In order to overpass the inversion difficulties generated by non-squared matrices, a pseudo-inverse matrix can be used,

$$
\mathbf{D}=\left(\mathbf{O}^{T} \mathbf{O}\right)^{-1} \mathbf{O}^{T}
$$

Based on this, the efficiency of the modulation is defined as:

$$
\epsilon_{i}=\left(N \sum_{j=1}^{N} \mathbf{D}_{i j}^{2}\right)^{-\frac{1}{2}} \quad i=1,2,3,4
$$

The total efficiency of the modulation scheme is also defined by

$$
\epsilon=\sqrt{\epsilon_{2}^{2}+\epsilon_{3}^{2}+\epsilon_{4}^{2}}
$$

with a maximum reachable efficiency of 1 .

Our previous research has proved that the efficiency of this modulator is high (0.99), placing the concept above classical cases such as ZIMPOL-Zurich Imaging Polarimeter (0.72), ASP - Advanced Stokes Polarimeter (0.88), or TIP - Tenerife Infrared Polarimeter (0.92). ${ }^{4,6,7}$ From equations (1), (2), (3), (9) it follows that the efficiency is impacted by four parameters: the angles $\xi$ and $\psi$ of the modulator, the angle $\theta$ of the analyzer, and the number of pixels. Analyzing the efficiency of the polarimeter as a function of these parameters, recommendations for the best geometry, the orientation of the analyzer, or the dimension of the detector can be inferred. Therefore, orientations of the analyzer at $45^{\circ}$ and $135^{\circ}$ should be avoided as they lead to an indetermination of the $Q$ parameter of the incoming light. Concerning the apex angles $\xi$ and $\psi$, values $\left(2.6^{\circ}, 1.6^{\circ}\right)$, for instance, are ensuring equal efficiency in the determination of $U$ and $V$. Simulations conducted for values of $\xi$ and $\psi$ between $1^{\circ}$ and $4^{\circ}$ show that for $\xi=\psi$ an important drop of efficiency is observed (Fig. 5). Values of the type $\xi=2 \psi$ or $\psi=2 \xi$ are also characterized by a certain drop of efficiency, the effect being more important when the apex angle of the second wedge is bigger than the first, $\psi>\xi$. The reason of this efficiency drop is the fact that the scalar product of the modulation functions is no longer zero if $\xi=\psi$ or $\psi=2 \xi$. The same situation can be pointed out for $\xi=2 \psi$. Consequently, the modulation functions are no longer orthogonal in these cases. Therefore the uniqueness of the solution is not verified for this configurations of the modulator and the uncertainty characterizing the Stokes parameters is maximal. Because of this, the geometry of the modulator should have:

$$
\left\{\begin{array}{l}
\psi \neq k \cdot \xi, \quad k=1,2, \ldots \\
\xi \neq k \cdot \psi
\end{array}\right.
$$


Concerning the impact of the number of pixels, it can be noticed that the maximum efficiency is recorded for at least 100 pixels if $\lambda=0.3 \mu \mathrm{m}$, this limit being dependent of the wavelength. Increasing the number of pixels above this value brings no further improvement for the measurement of polarization at this wavelength.
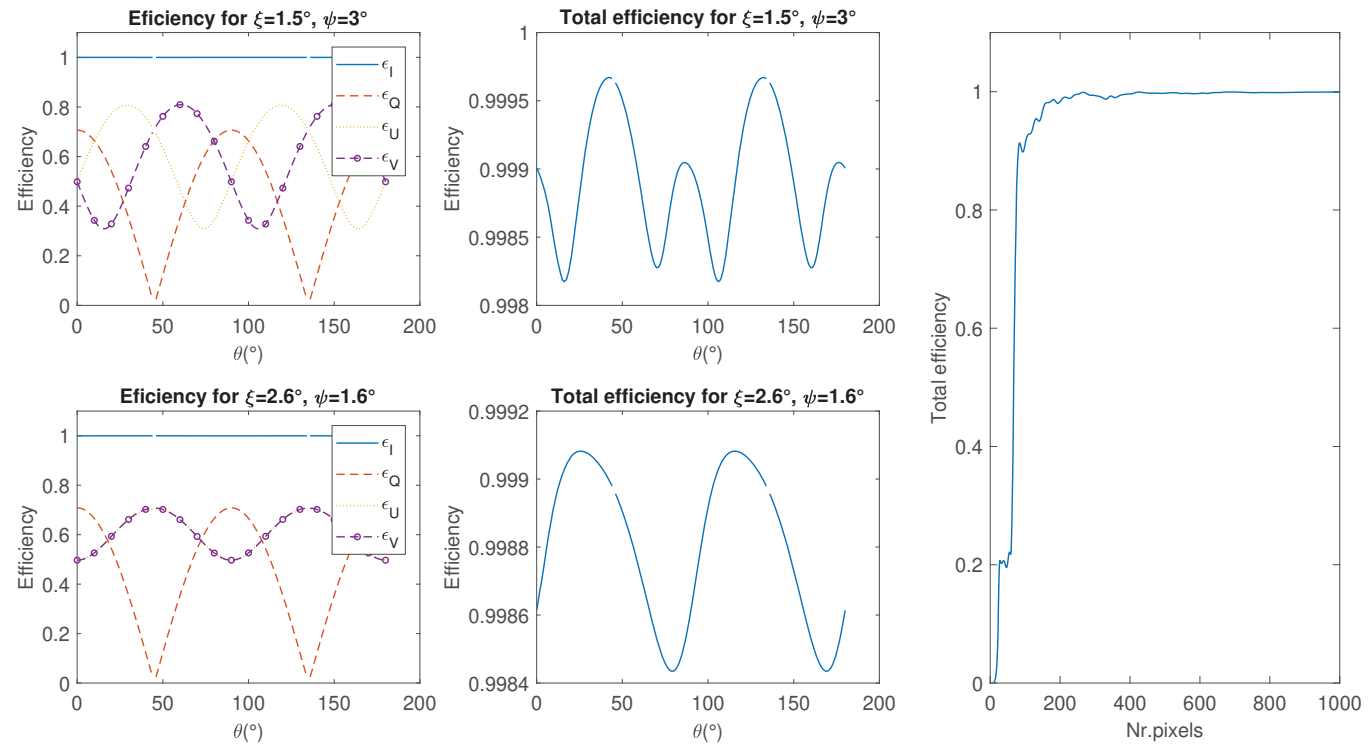

Figure 4. Efficiency of the modulation scheme and total efficiency as a function of the orientation of the analyzer $(\theta)$, for two couples $(\xi, \psi):\left(1.5^{\circ}, 3^{\circ}\right)$ and $\left(2.6^{\circ}, 1.6^{\circ}\right)$. The total efficiency as a function of the number of pixels is also plotted (right). The variations with $\theta$ are considered for the case of 1000 pixels of $10 \mu \mathrm{m}$ dimension. The efficiency as a function of number of pixels is observed for the scenario $\xi=2.6^{\circ}, \psi=1.6^{\circ}, \theta=30^{\circ}$, and $\lambda=0.3 \mu \mathrm{m}$. 

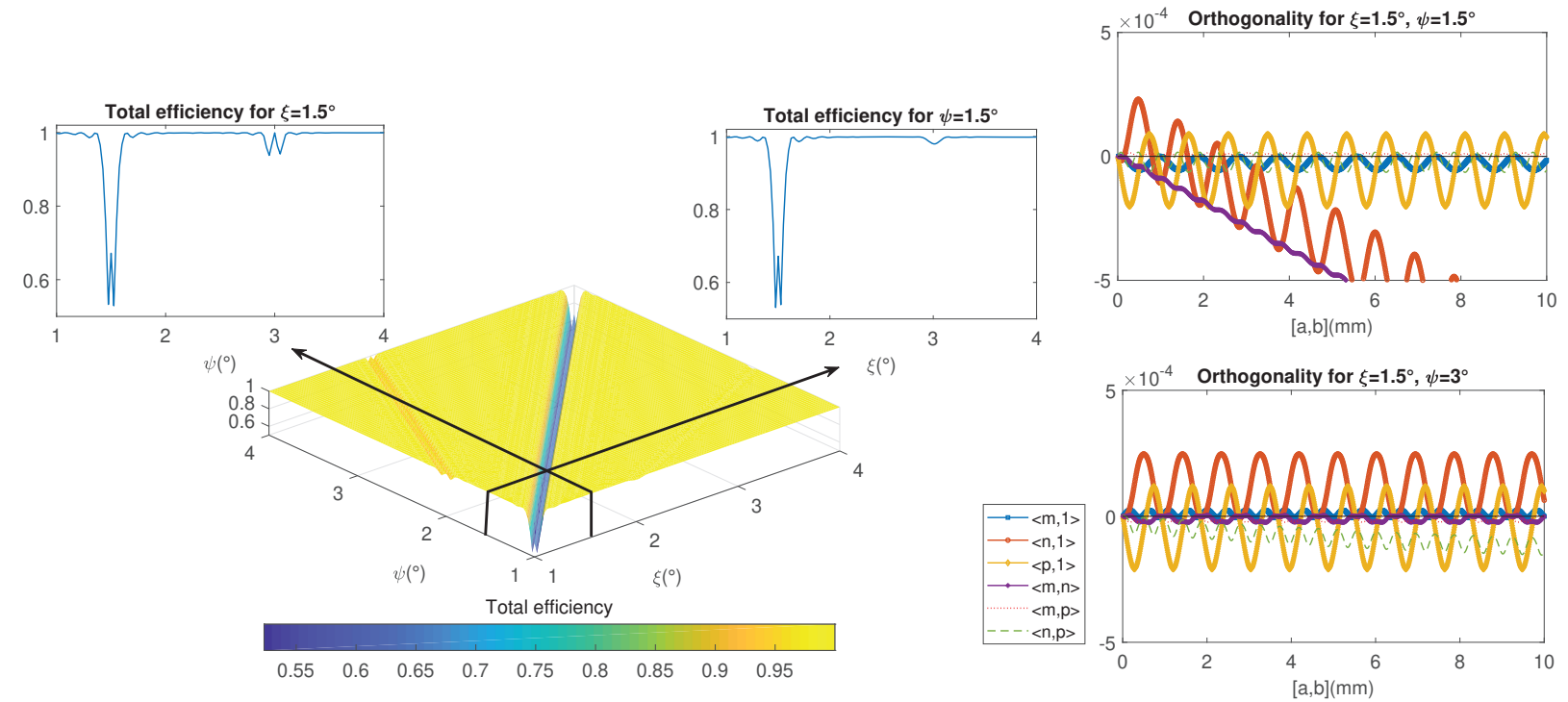

Figure 5. Total efficiency as a function of $\xi$ and $\psi$ angles of the modulator. The contour plot shows that equal values, $\xi=\psi$, or situations of the type $\xi=2 \psi$, respectively $\psi=2 \xi$, should be avoided as they generate a drop of efficiency. The particular cases of $\xi=1.5^{\circ}$ and $\psi=1.5^{\circ}$ are detailed. At right, the scalar product of the modulation functions for the particular cases $\xi=\psi=1.5^{\circ}$, and $\psi=2 \xi=3^{\circ}$ is represented. It is noticeable the high slope of $\langle m, n>$ and $\langle n, 1>$ in the case $\xi=\psi=1.5^{\circ}$, characterized also by a huge drop of efficiency, and the smaller deviation of $\left\langle n, p>\right.$ when $\xi=1.5^{\circ}$, $\psi=3^{\circ}$, corresponding also to a local minimum of efficiency.

\section{CONCLUSION}

A new concept of spectropolarimeter was introduced in this short and mostly theoretical description. The key part of the instrument, the modulator, presents a stable triple prismatic structure and is build from a uniaxial birefringent material. A new and rapid method for demonstrating the uniqueness of the solution was also presented. This is based on the demonstration of the orthogonality of the modulation functions. Previously, the proof for the uniqueness was founded on the independence of the modulation functions. ${ }^{4}$ This can be revealed through the calculation of the Wronskian, method which is very compute-intensive. The particular geometry of this new modulator enables very high efficiency of the modulation scheme and therefore a high accuracy of measurements. Simulations have been conducted for various geometries and show that an almost perfect working mode is reachable, placing the concept above classical examples of spectropolarimeters. A complete mapping of the efficiency as a function of the main parameters of the polarimeter was presented here. A careful analysis of the efficiency behavior in function of the geometrical configuration enables a precise determination of the configurations with the highest potential. Simultaneously, architectures that may enhance the precision in the measurement of one or another of the Stokes parameters can be pointed out.

\section{REFERENCES}

[1] Bailey, J., Kedziora-Chudczer, L., and Bott, K., "Polarized radiative transfer in planetary atmospheres and the polarization of exoplanets," Mon. Not. R. Astron. Soc. 480(2), 1613-1625 (2018).

[2] Rossi, L. C. G. and Stam, D. M., "Circular polarization signals of cloudy (exo)planets," Astron. Astrophys. 616, A117 (2018).

[3] Kolokolova, L., Hough, J., and Levasseur-Regourd, A. C., [Polarimetry of Stars and Planetary Systems], Cambridge University Press (2015).

[4] Vasilescu, B., Nazé, Y., and Loicq, J., "Solution uniqueness and noise impact in a static spectropolarimeter based on birefringent prisms for full Stokes parameter retrieval," J. Astron. Telesc. Instrum. Syst. 6, 1 (Apr. 2020). [doi:10.1117/1.JATIS.6.2.028001]. 
[5] del Toro Iniesta, J. C., [Introduction to spectropolarimetry], Cambridge University Press, Cambridge (2003).

[6] del Toro Iniesta, J. C. and Collados, M., "Optimum modulation and demodulation matrices for solar polarimetry," Appl. Opt. 39, 1637-1642 (Apr 2000).

[7] Collados, M., "High resolution spectropolarimetry and magnetometry," in [3rd Advances in Solar Physics Euroconference: Magnetic Fields and Oscillations], B.Schmieder, A.Hofmann, J., ed., ASP Conference Series 184 (1999). 